\title{
Investigation of a confined C-class flare in an arch filament system close to a regular sunspot
}

\author{
Rohan Eugene Louis \\ ${ }^{1}$ Udaipur Solar Observatory, Physical Research Laboratory, Dewali Badi Road, Udaipur - 313001, \\ Rajasthan, India \\ manuscript-v3.tex compiled on October 15, 2019 \\ Received 2019 March 01; Revised 2019 July 23; Accepted 2019 September 23
}

\section{Key Points:}

- C1.1 class confined flare occurred in an arch filament system close to a regular, unipolar sunspot on 2013 September 24

- Flare driven by small-scale, flux cancellation at neutral line underlying arch filament increasing filament twist and destabilizing it

- Twisted filament expands as it rises producing 2-ribbon flare at neutral line but confined by overlying horizontal fields of active region

Corresponding author: Rohan Eugene Louis, rlouis@prl.res.in 


\begin{abstract}
A moderate C1.1 class confined flare is investigated here, which occurred on 2013 September 24 at 22:56 UT, in an arch filament system close to a regular, unipolar sunspot. Spectropolarimetric observations from the Tenerife Infrared Polarimeter at the $70 \mathrm{~cm}$ German Vacuum Tower Telescope were combined with data from the Helioseismic Magnetic Imager and the Atmospheric Imaging Assembly to identify the processes that triggered the flare. The legs of this arch filament were anchored in the leading sunspot and the network flux region of opposite polarity. The flare was driven by small-scale, flux cancellation at the weak neutral line underlying the arch filament which resulted in two small flaring events within an hour of the C1.1 flare. Flux cancellation was facilitated by the moat flow from the leading sunspot wherein small-scale magnetic fragments stream towards patches of pre-existing flux. The cancellation of flux led to the destabilization of the arch filament which was seen as an increase in the twist along the arch filament. The horizontal fields across the weak neutral line decay faster which cannot prevent the filament from rising that results in a two-ribbon flare at the neutral line. The arch filament unwinds as it rises, but is confined by the higher, overlying fields between the two polarities of the active region that decay much more slowly.
\end{abstract}

\title{
1 Introduction
}

Solar flares are energetic phenomena that result in particle acceleration, plasma heating, and the release of energy over the entire electromagnetic spectrum. Flares are characterized by a sudden onset and develop on time scales that are shorter than the photospheric evolution time scale. Photospheric motions are responsible for storing magnetic energy in the corona which is then impulsively released at a critical point in the evolution of the coronal field (Forbes, 2010), resulting in a flare. Flares that are associated with coronal mass ejections (CMEs) (Gosling, 1993; Kahler, 1992; Plunkett et al., 2000) are termed eruptive and tend to occur in regions of a highly non-potential coronal field, that overlie the photospheric polarity inversion lines (Hagyard, Venkatakrishnan, \& Smith, 1990; H. Wang, 2006). While it has been shown that a large majority of X-class flares are associated with CMEs (Yashiro, Akiyama, Gopalswamy, \& Howard, 2006) and tend to occur in active regions with more free energy (Cui, Wang, Xu, \& Liu, 2018), flares can be non-eruptive or confined, failing to produce a CME. A prominent example of confined flares are those associated with the largest active region (AR) of solar cycle 24, NOAA AR 12192. This AR was flare-rich but CME-poor, producing 6 X-class flares, 22 M-class flares, and 53 C-class, but only 1 CME which resulted from an M4.0 class flare (Chen et al., 2015; C. Jiang et al., 2016; L. Liu et al., 2016; Sarkar \& Srivastava, 2018; Sun et al., 2015). Cui et al. (2018) analyzed 58 X-class flares that occurred within $30^{\circ}$ of disk center between 1996 to 2005 and found that 10 X-class flares were confined.

The source regions of CMEs show a filament channel in the chromosphere (Karpen, Antiochos, \& DeVore, 2012), which is a region of strong magnetic non-potentiality confined around the polarity inversion line (PIL). The filament channel could eventually harbor a filament or prominence, wherein the $\mathrm{H} \alpha$ fibrils are aligned with the PIL (Foukal, 1971; Martin, 1998a). While a large majority of erupting filaments or prominences are closely associated with flares, their association with CMEs is about 50\% (Jing, Yurchyshyn, Yang, Xu, \& Wang, 2004; Yan, Qu, \& Kong, 2011). Filament eruptions, flares, and CMEs are evidences for the destabilization of the coronal magnetic field and their association is still an open ended topic (Schrijver, 2009).

Unlike, PIL filaments, arch filament systems (AFS) are a bunch of dark filament threads crossing the PIL and connecting the regions of opposite polarity, sometimes extending into sunspots (Bruzek, 1967; Weart \& Zirin, 1969; Zwaan, 1985). The arches in the AFS exhibit an upward motion of about $10-15 \mathrm{~km} \mathrm{~s}^{-1}$ and downwflows of about $10-50 \mathrm{~km} \mathrm{~s}^{-1}$ along their legs. Flares occurring in AFS arise from reconnection with pre-existing magnetic fields (Huang et al., 2018; Zuccarello et al., 2008) or through persistent flux cancellation (Su et 
al., 2018; Y. Wang et al., 2018). In this article, I investigate a moderate C1.1 class, confined flare, which occurred in an AFS outside a regular, isolated sunspot on 2013 September 24. The photospheric and coronal conditions are analyzed in order to determine what triggered the flare, in the otherwise quiet and small active region, and rendered it confined.

\section{Observations and data analysis}

I carried out spectropolarimetric observations of NOAA AR 11846 on 2013 September 24 using the Tenerife Infrared Polarimeter (TIP-II; Collados et al., 2007) at the $70 \mathrm{~cm}$ German Vacuum Tower Telescope (VTT), Observatorio del Teide, Tenerife, Spain. TIP II provided spectral scans with full-Stokes polarimetry in the near infrared region comprising the Fe I line at $10783.05 \AA$, and the Si I line at $10786.85 \AA$. Both lines have a Landé $g$ factor of 1.5 and an excitation potential of $3.06 \mathrm{eV}$ and $4.93 \mathrm{eV}$, respectively (Balthasar \& Gömöry, 2008).

The AR consisted of a unipolar leading sunspot located at at a heliocentric angle of $26.6^{\circ}\left(143^{\prime \prime},-404^{\prime \prime}\right)$. At each slit position and for a single modulation state, ten exposures of $250 \mathrm{~ms}$ each were accumulated. Using a $0^{\prime \prime} .35$-wide slit, a full scan of the sunspot was carried out with 160 scan steps, each step being 0". 35. The scan lasted from 9:53-10:26 UT. The spatial sampling along the slit was 0 "' 17 while the spectral sampling was $11.05 \mathrm{~m} \AA$. A dichroic beam splitter plate, placed in the adaptive optics tank on the first floor of the VTT, was used to carry out the scan. A small fraction of light from the beam splitter was transmitted to the wave-front sensor (WFS) while the rest of the light was reflected to the science focus. A small tilt shifted the image at the science focus but not on the WFS. Flat-fielding and polarization calibration were performed at the end of the scan, using a rotatable linear polarizer and a phase plate which were introduced in the optical path after the exit window of the vacuum tower (Collados, 1999; Louis, Balthasar, et al., 2014). The observations were supported by the Kiepenheuer-Institut Adaptive Optics System (KAOS; von der Lühe, Soltau, Berkefeld, \& Schelenz, 2003) which provided a highly stable scan of the sunspot with a spatial resolution of about $1^{\prime \prime}$. Figure 1 shows the TIP-II observations of the leading sunspot in AR 11846 along with the Stokes spectra spanning the near infrared region at $1078 \mathrm{~nm}$.

The vector magnetic field and other relevant physical parameters were extracted from the TIP-II spectro-polarimetric observations using the Stokes Inversion based on Response Functions (SIR; Ruiz Cobo \& del Toro Iniesta, 1992) code. SIR computes perturbations in the physical quantities at specific locations across the optical depth grid called nodes, and then carries out an interpolation to yield values at all grid points. I performed a onecomponent inversion setting the magnetic and dynamic parameters to be constant with depth and inverted the Fe and Si lines separately since the lines form in a narrow region of the photosphere. This is similar to the strategy adopted by Balthasar and Gömöry (2008). The temperature stratification was perturbed with five nodes. A total of nine parameters were retrieved from the observed profiles, including height-independent micro- and macroturbulent velocities. The vector magnetic field was transformed to the local reference frame from the line-of-sight (LOS) frame. The $180^{\circ}$ ambiguity in the azimuth was resolved by assuming a radial variation of the horizontal magnetic field and a smooth azimuth (Louis, Beck, \& Ichimoto, 2014).

I also combined the TIP observations with those from the Helioseismic and Magnetic Imager (HMI; Schou et al., 2012) as well as the Atmospheric Imaging Assembly (AIA; Lemen et al., 2012). The HMI data consist of continuum intensity filtergrams and line-ofsight (LOS) magnetograms having a cadence of $45 \mathrm{~s}$, while the AIA observations comprise filtergrams in the He II $304 \AA$ and Fe IX $171 \AA$ bands having a cadence of 1 min. The HMI and AIA data were selected to span a duration of $3 \mathrm{hr}$ starting at 21:00 UT leading up to the C1.1 flare at 22:56 UT. The HMI and AIA images were co-aligned using a two-dimensional 

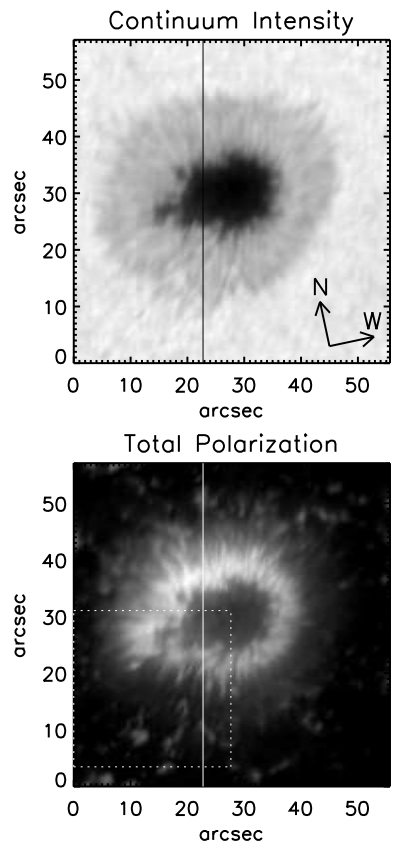
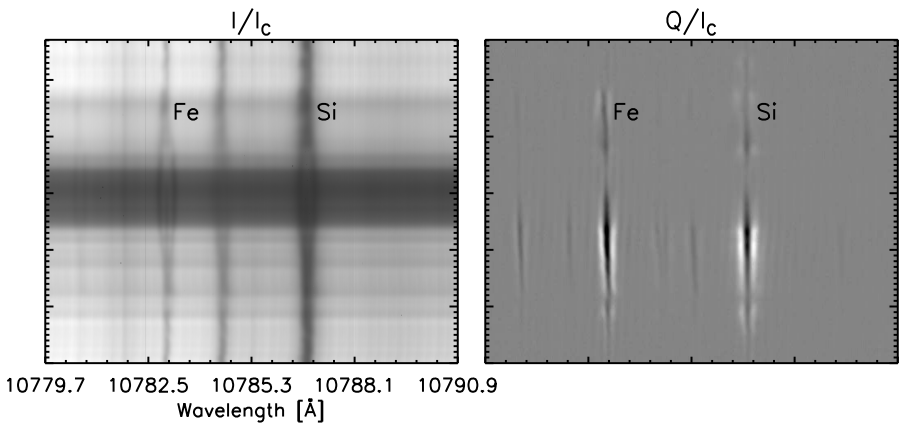

$\mathrm{U} / \mathrm{I}_{\mathrm{c}}$
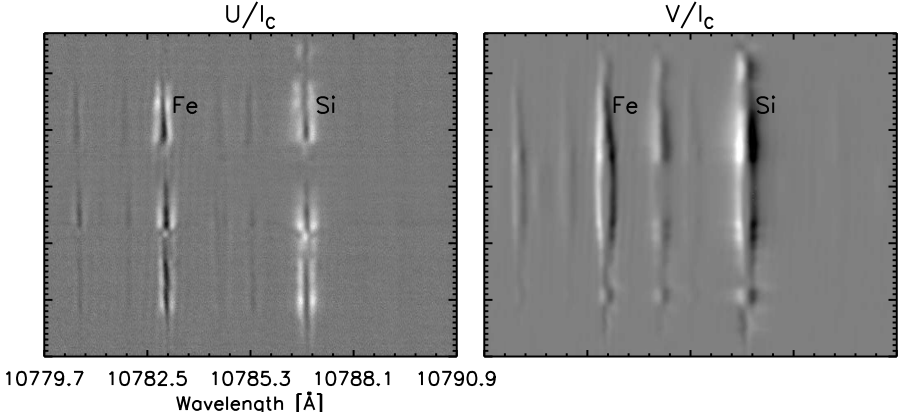

Figure 1. Near infrared spectropolarimetric observations using TIP-II. The top left panel shows the continuum intensity at $10780 \AA$. The orientation of the TIP data is indicated in the bottom right corner. The bottom left panel shows the map of the total polarization derived from the Si line. The white dotted box corresponds to the field-of-view shown in Fig. 7. The middle panels represent the spectral window containing the Fe and Si lines, in Stokes $I$ (top) and $U$ (bottom), respectively, while the right panels correspond to Stokes $Q$ (top) and $V$ (bottom), respectively. The Stokes spectra correspond to the vertical cut shown in the left panels.

cross correlation routine. AIA images with a cadence of $12 \mathrm{~s}$ were also utilized close to the time of the precursor events as well as the C1.1 flare.

\section{Results}

\subsection{General configuration}

Figure 2 shows NOAA AR 11846 to consist of a single, regular, leading sunspot of positive polarity with a diffused network region, about $90^{\prime \prime}$ to its east, which is the following polarity of the AR. The network region only shows up in the continuum image as a couple of tiny pores. The AR appeared close to the Sun's eastern limb on 2013 September 18 and for a major part of its transit on the disc, remained an $\alpha$-type spot. It finally disappeared at the western limb during the early half of September 30. During its passage, the AR produced three flares, a C3.9 class flare at 03:15 UT on September 18, a B4.8 class flare at 21:54 UT on September 23, and a C1.1 class flare at 22:56 UT on September 24.

The lower panels of Fig. 2 show the chromosphere and transition region above the AR. The AIA $304 \AA$ image (bottom left panel of Fig. 2) reveals the presence of superpenumbral whirls around the AR having a distinct handedness, synonymous with a sinistral chirality (Chae, 2000; Martin, 1998b, 2003) that is consistent with the helicity hemispherical rule. The image also shows three AFS (marked F1, F2 and F3) all of which originate from the network patch and terminate at/close to the boundary of the leading sunspot. While F1 and F3 are quite distinct, F2 is extremely faint and is more clearly seen as a bright, thread-like structure in the AIA $171 \AA$ image (bottom right panel). These chromospheric AFS have 

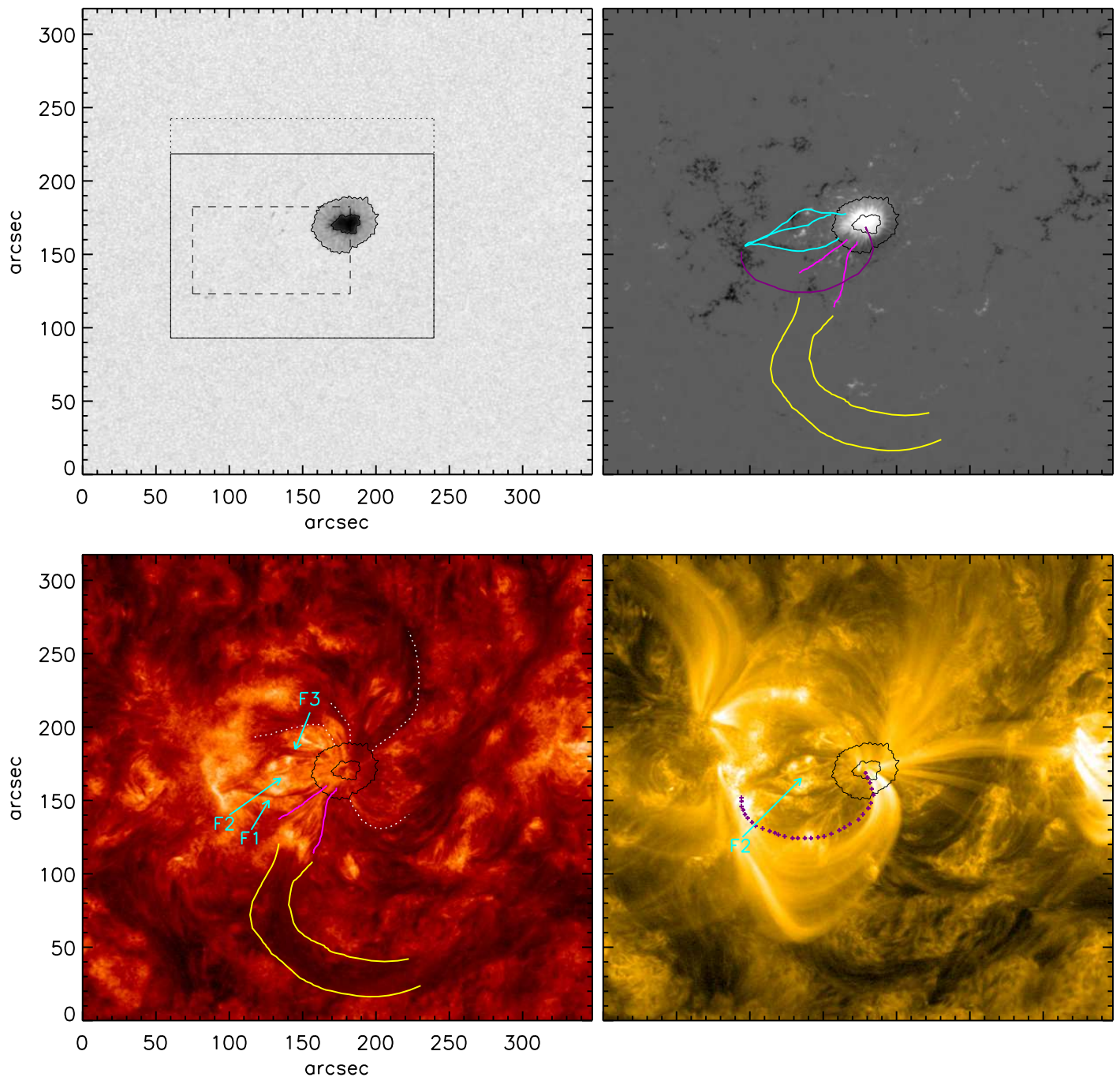

Figure 2. Top panels: HMI continuum image (left) and LOS magnetogram (right) of NOAA AR 11846 on 2013 September 24 at 21:00 UT. The dashed, dotted, and solid lines in the top left panel correspond to the field-of-view shown in Figs. 3, 5, and 6, respectively. Bottom panels: AIA $304 \AA$ image (left) and $171 \AA$ image (right). The white dotted lines in the lower left panel indicate superpenumbral whirls of the active region with a clockwise orientation. The black contours outline the umbra-penumbra and penumbra-quiet Sun boundary. North is up and East is to the left. The colours in the bottom panels indicate different features (see text) and the same have been overlaid on the HMI LOS magnetogram.

been marked since the flare occurred in this part of the AR, specifically involving F1. For the sake of brevity and clarity, I will henceforth refer to the AFS simply as filaments in the text, while the term PIL filaments will be used to distinguish the AFS from regular, chromospheric filaments.

The projected lengths of the filaments F1, F2, and F3 was estimated to be 49, 46, and $56 \mathrm{Mm}$, respectively. In addition, there exists a large filament channel south of the leading sunspot which also exhibits a sinistral chirality (shown in yellow in the figure). Hereafter this feature will be referred to as the dark channel since there are no clear signatures of an extended PIL filament in $\mathrm{H} \alpha$ (https://gong2.nso.edu/products/scaleView/ 
view.php? configFile=configs $/$ hAlpha.cfg\&productIndex=1). The dark channel starts from a diffuse patch of negative polarity flux south-east of the sunspot. Short, dark fibrils (shown in magenta in the figure) extend from this patch to the outer penumbra of the sunspot. The bottom right panel of Fig. 2 shows the structure of the AR in the transition region as seen in the AIA $171 \AA$ image and indicates several, bright loops connecting the leading sunspot to the network patch in addition to the filaments seen in the chromosphere. The lowest of these loops is highlighted in purple in the figure and is estimated to lie about $24 \mathrm{Mm}$ above F1. The highest of the AIA loops connecting the leading sunspot to the network patch are at a projected height of $100 \mathrm{Mm}$ with respect to F1. Apart from these set of loops, one observes connections between the sunspot and opposite polarity flux lying to the north-east and west of the former. To the west of the sunspot there is another PIL filament that is visible in both AIA bands which has the same handedness as the superpenumbral whirls associated with the AR. This feature is also seen in $\mathrm{H} \alpha$ unlike the dark channel described above. The filaments F1, F2, and F3, as well as the overlying loops identified in the AIA 304 and $171 \AA$ images, respectively, are overlaid on the HMI magnetogram with their corresponding colors to provide a context of the magnetic topology of the AR. The chromospheric and transition region features identified in Fig. 2 and described above correspond to a time close to the occurrence of the flare and nearly $12 \mathrm{hrs}$ after the TIP-II observations. However, these features are long-lived, with the exception of filament F2, and are seen to be present well before the TIP-II observations, which puts their base lifetime at $12 \mathrm{hrs}$.

\subsection{Small-scale flaring activity}

In this section, two examples of small-scale flaring activities in the AR, that occur within one hour of the C1.1 flare, are described. In addition to these events, the AR is also characterized by several other dynamic phenomena, which include brightenings along the short dark fibrils (indicated in magenta in Fig. 1) south east of the leading sunspot, and filamentary ejections that start at the location of the diffuse polarity south of the leading sunspot and travel southward along the dark channel(These can be seen in the animation movie-03.avi that is available as supplementary material). Along with the AIA $171 \AA$ band, these precursor events are also seen in the hotter 193 and $211 \AA$ channels but not in the 335 and $94 \AA$ bands, that would suggest that the plasma is heated to at least $10^{5} \mathrm{~K}$.

\subsubsection{Event 1}

Figure 3 shows a sequence of AIA $304 \AA$ and $171 \AA$ images centered on the filament F1. Panels 1 and 2 indicate that there is a diffuse, thin, loop-like structure which struts out of F1 extending from the eastern end to the central part of F1. The white arrow in panel 2 shows the structure much more clearly using a base difference image. At 21:55:31 UT, a similar set of loops are seen to unravel from F1, expanding rapidly southwards as shown by the black arrow in panels 3-5. This unfurling lasts for about $1 \mathrm{~min}$ at the end of which remote brightenings are seen at the footpoints indicated by the black circles in panel 6 . The larger brightening at F1, closer to the leading sunspot, appears at 21:57:31 UT, about a $1 \mathrm{~min}$ before its counterpart which appears on the southern ridge of F1 close to the network flux region. The small flaring activity lasts for about $2.5 \mathrm{~min}$ and is analogous to the event described in H. Wang et al. (2015).

The underlying photospheric conditions related to the above flaring event is shown in Fig. 4 where there are clear indications of flux cancellation at the location of the footpoint brightening. The reduction in flux is more apparently seen in the negative polarity fragment that disappears at the interface of the positive polarity fragment outlined by the dark blue contour. The decrease in magnetic flux is about $9.8 \times 10^{17} \mathrm{Mx}$ and $6.3 \times 10^{17} \mathrm{Mx}$ in the positive and negative polarity patches, respectively and occurs over a duration of about 37 min which yields a flux loss rate of $4.5 \times 10^{14} \mathrm{Mx} \mathrm{s}^{-1} 2.9 \times 10^{14} \mathrm{Mx} \mathrm{s}^{-1}$, respectively. The HMI LOS magnetogram has been overlaid on the AIA $304 \AA$ image for an overall perspective, that shows the filament F1 arching from the leading sunspot to the network 

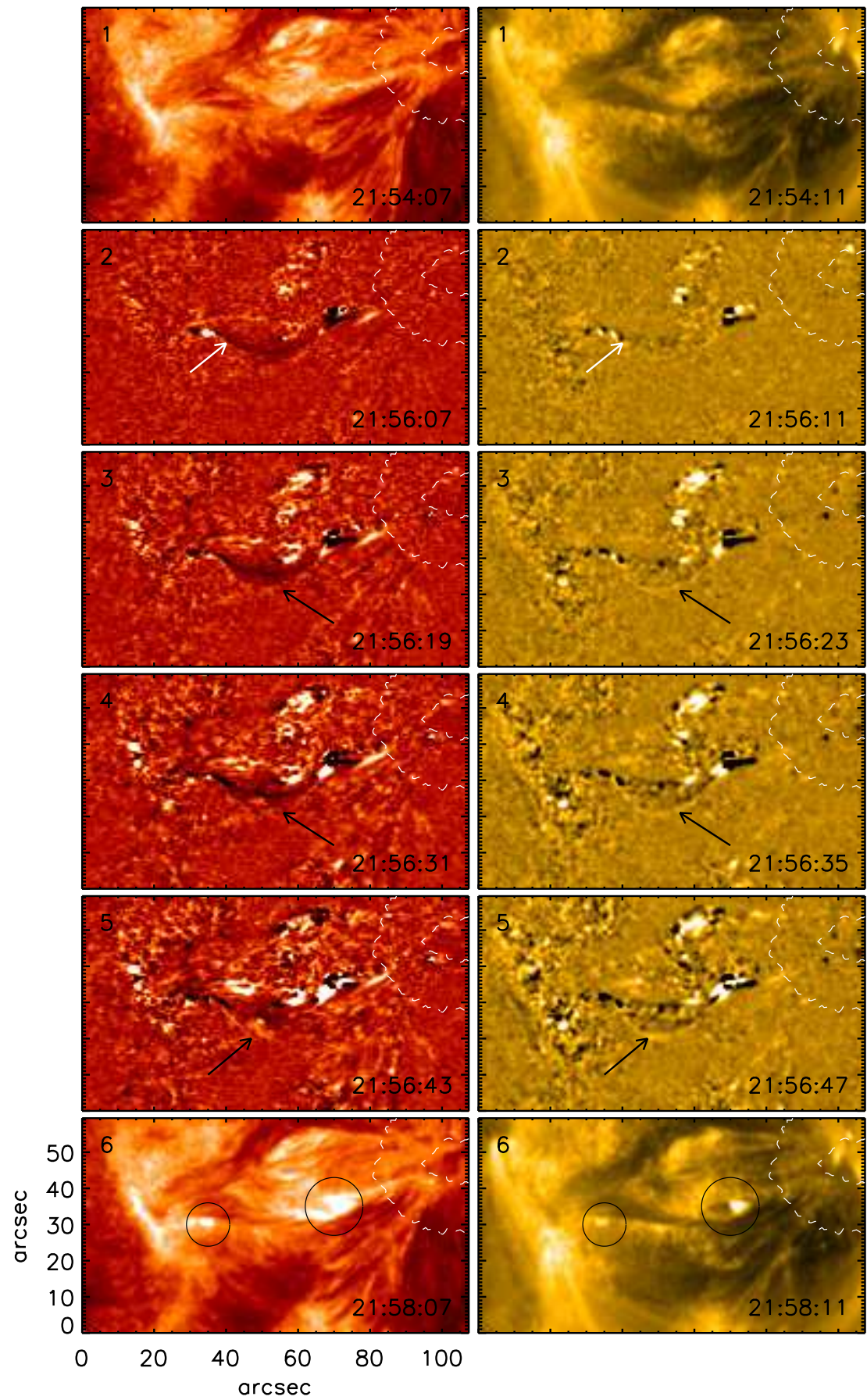

Figure 3. Time sequence depicting the pre-flaring activity in F1. The left and right columns correspond to the AIA $304 \AA$ and $171 \AA$ channels, respectively. The white and black arrows indicate a set of loops that expand rapidly and detach from the filament F1, producing small-scale brightenings at the footpoints of the loops enclosed in the black circle indicated in panel 6 . The images in panels 2 to 5 are base difference images using a reference image at 21:55:55 UT. An animation of the figure (movie-01.avi) is available as supplementary material.

flux region with a smaller, extended flux patch of negative polarity under the axis of F1. 


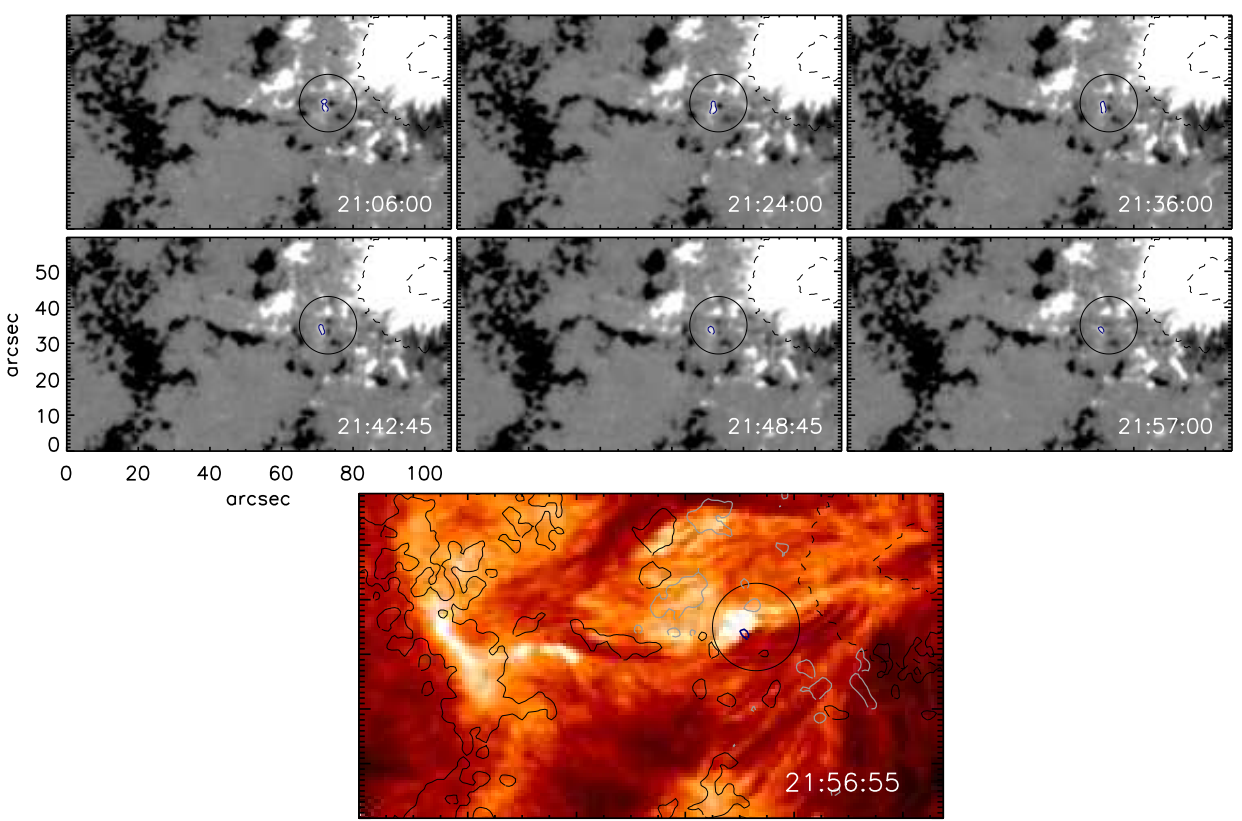

Figure 4. Flux cancellation event (black circle) leading up to the flaring activity shown in Fig. 3. The dark blue contour outlines a positive polarity patch which indicates the cancellation of a neighboring magnetic fragment of opposite polarity. The bottom panel shows an AIA $304 \AA$ image with the one of the footpoint brightenings occurring at the location of flux cancellation. The black and grey contours correspond to negative and positive polarity, respectively at a value of $\pm 100 \mathrm{G}$. The dashed black lines outline the umbra-penumbra and penumbra-quiet Sun boundary of the leading sunspot.

The general picture is that F1 overlies a weak PIL with the footpoint brightenings from the precursor event located on either side of this PIL.

\subsubsection{Event 2}

The second event that was analyzed occurs in nearly the same location as described above and is also driven by flux cancellation. The left panels of Figure 5 show a similar flaring activity as earlier at 22:33 UT, nearly 30 min after the first event. A comparison of panels 1 and 2 in the figure shows that the F1 to exhibit twisted, dark and bright and threads (dotted line in panel 2), with the bright thread running under its dark counterpart. This intersection point coincides with a small localized brightening which is essentially at the weak PIL under F1. The crossing of the bright and dark threads as seen in panel 2, would suggest that the a twist of least half a turn is present in F1. The stronger brightening near the central part of $\mathrm{F} 1$ peaks about $30 \mathrm{sec}$ before its counterpart at the southern ridge of F1 (panels 3 and 4). The flare is immediately followed by an injection of cooler plasma along the eastern section of $\mathrm{F} 1$ as indicated by the black arrows in panels 5 and 6 .

The right panel of Fig. 5 shows the time sequence of LOS magnetograms that coincide with the second flaring event. The cancellation is primarily seen in the right most patch of positive polarity outlined by the red contour. There are only three small fragments of positive polarity nearest the larger patch of negative polarity that sits under the central part of F1. The flux reduction in these three patches from left to right are $3.9 \times 10^{17} \mathrm{Mx}$, $9.7 \times 10^{17} \mathrm{Mx}$, and $8.8 \times 10^{17} \mathrm{Mx}$, respectively over a duration of about $28 \mathrm{~min}$. Using the above values the flux loss rate is estimated to be $2.4 \times 10^{14} \mathrm{Mx} \mathrm{s}^{-1}, 5.8 \times 10^{14} \mathrm{Mx} \mathrm{s}^{-1}$, and $5.3 \times 10^{14} \mathrm{Mx} \mathrm{s}^{-1}$, respectively. While there is a substantial reduction in positive flux, the 

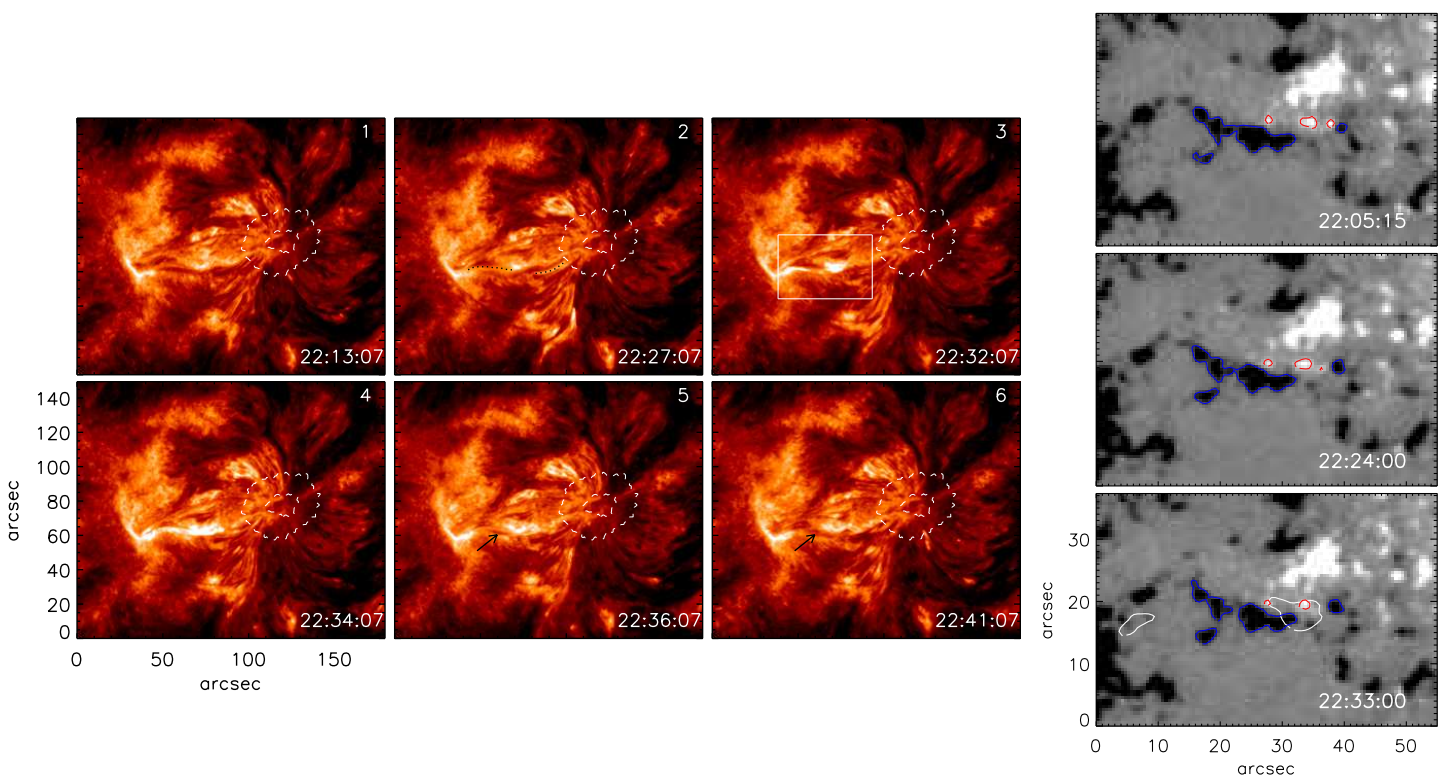

Figure 5. Left: AIA $304 \AA$ images depicting another small flaring event that occurred prior to the C1.1 flare. The black dotted line in panel 3 shows a bright thread crossing under a dark thread. The white box in panel 3 corresponds to the field-of-view shown on the right. The arrows in panels 5 and 6 indicate the injection of cool plasma into the filament F1 after the flare. Right: Time sequence of LOS magnetograms showing flux cancellation occurring close to the weak PIL under the mid-section of F1 leading to the event shown on the left. The blue and red contours correspond to negative and positive polarity, respectively at a value of $\pm 100 \mathrm{G}$. The white contours in the bottom right panel correspond to the footpoint brightenings. An animation of this figure is available as supplementary material.

right most patch of negative polarity, where the disappearance of the opposite polarity is distinct, exhibits an increase of flux amounting to $2.6 \times 10^{18} \mathrm{Mx}$. The larger fragment of negative polarity on the left outlined by the blue contours, however, shows a reduction in flux of about $2.4 \times 10^{17} \mathrm{Mx}$. The location of the disappearance of the positive flux is about $10^{\prime \prime}$ east of the cancellation event described in Sect. 3.2.1. It is also evident from the bottom panel that the small-scale changes in photospheric flux are co-spatial with the stronger footpoint brightening seen at the intersection of the bright and dark filament threads of F1.

\subsection{The C1.1 flare in F1}

The precursor flare at 22:33 UT results in the buildup of cool plasma along the filament F1 (panel 6 of Fig. 5). This process continues for about 10 min when filamentary brightenings appear at the ends of the filament F1, i.e. at the outer penumbra of the leading sunspot and the network flux region of opposite polarity and are indicated by cyan ellipses in panel 1 of Fig. 6. The figure also shows that there is an enhancement in the twist along F1 which are depicted by black arrows in the figure and can be compared with the filament structure nearly 20 min earlier (panel 2 of Fig. 5). The filamentary brightening at the eastern end of F1 intensifies by 22:48 UT which is accompanied by an untwisting of F1 at that location in the counterclockwise direction as seen along the filament axis from the leading sunspot to the following polarity (arrow in panel 2). The unwinding of F1 is accompanied by an increase in the emission of the filament that expands southwards as F1 rises (panels 3-5). However, panel 6 also reveals that along with the rise of F1, the flaring occurs along the weak PIL under F1 where small-scale flux cancellation was observed earlier, forming a classic 


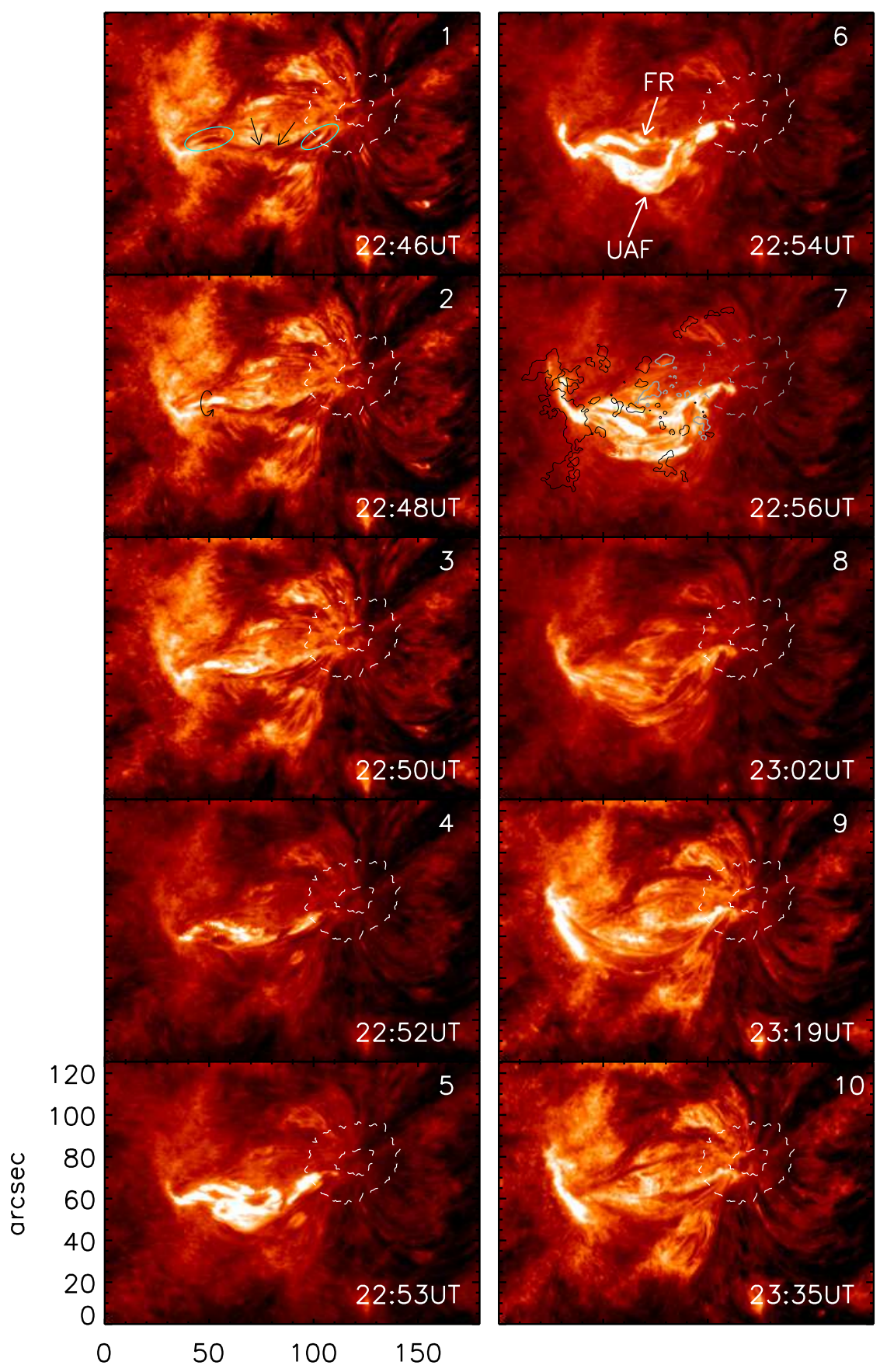

Figure 6. Evolution of the C1.1 flare seen in AIA $304 \AA$ images. The cyan ellipses in panel 1 indicate the onset of the flare with brightenings along threads that twist around the axis of F1. The black arrows in the panel indicate an enhancement in the twist in F1. Panels $4-8$ have been scaled with a higher intensity threshold to show the fine structure of the expansion of F1 as it flares. In panel 6, 'FR' and 'UAF' correspond to flare ribbon and untwisting arch filament, respectively. Panel 7 shows contours of the line-of-sight magnetic field overlaid on the AIA image with black and grey colors corresponding to negative and positive polarity, respectively.

two-ribbon structure. The C1.1 flare peaks at 22:55 UT with the flare brightening extending along F1 to the network flux region in the east, to the umbra-penumbra boundary of the 
leading sunspot. The LOS magnetogram contours have been overlaid on the AIA image in panel 7, that show the 2-ribbon emission on either side of the neutral line. The flare lasts for a duration of $13 \mathrm{~min}$ ending at 23:03 UT. The emission associated with the flare was located at a distance of about $20 \mathrm{Mm}$ and $23 \mathrm{Mm}$ from the flux weighted centroid of the active region, at the beginning and peak time of the event, respectively. Panels 9 and 10 of the figure show that the filament structure resembles a simple arch with cool plasma suspended between the two polarities of the AR. A shorter section of the filament is also reformed at the boundary of the leading sunspot near one of the footpoints of the overlying arch. The flare morphology seen in the AIA $304 \AA$ band is similar in the $171 \AA$ channel, with the higher lying set of loops, described in Fig. 1, remaining unaltered during the duration of the flare and are only weakly displaced southward by about $1 \mathrm{Mm}$ during the expansion of the filament.
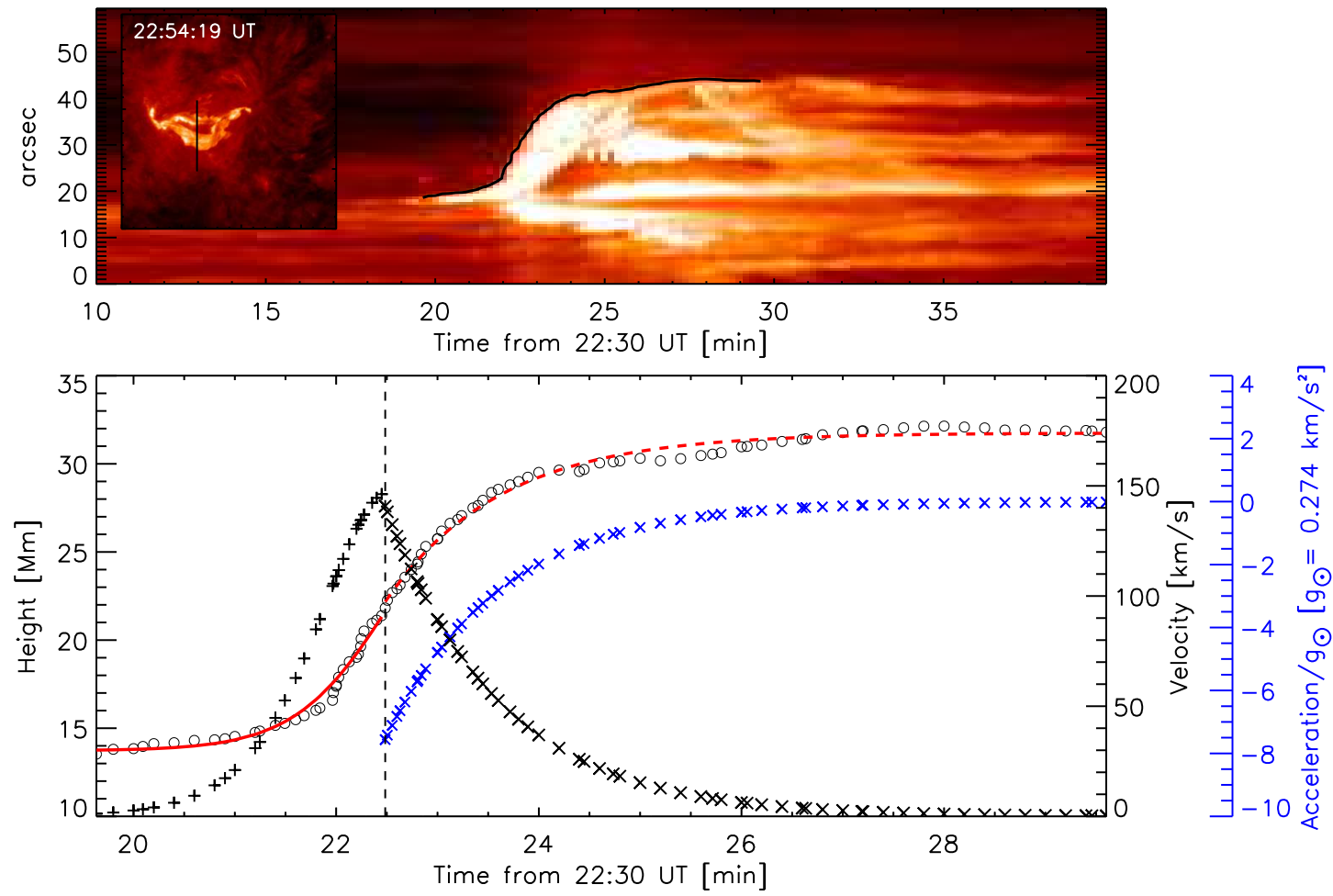

Figure 7. Kinematics of the rising filament during the flare. Top panel: space-time plot along the cut indicated by the black line in the inset at the top left corner. The black contour outlines the rising filament and is shown in the bottom panel as open circles. The space-time plot has been reversed in the vertical direction. Bottom panel: The red lines indicate the fit to the heighttime profile during the acceleration (continuous red line) and deceleration (red dashed line) phases, respectively, with the values indicated on the left $y$-axis. The corresponding velocity profiles during the two phases are shown as plus and cross symbols, respectively with the scale on the right $y$-axis. The deceleration profile is illustrated by bluecrosses whose scale is shown on the right. The vertical dashed line separates the acceleration and deceleration phases.

In order to estimate the speed of the expanding filament during the flare, a space-time plot was created as shown in Fig. 7. The inset in the top panel of the figure shows the position of the cut with the space-time plot reversed in the vertical direction for the sake of clarity. The height-time profile was extracted from the black contour outlining the top of the erupting filament which attained a maximum height of about $18 \mathrm{Mm}$ as indicated in the bottom panel of Fig. 7. The maximum velocity reached by the top of the filament 
is about $150 \mathrm{~km} \mathrm{~s}^{-1}$ at about 22:52:29 UT as shown by the plus symbols in the figure. When the flare peaks at 22:55 UT, the filament had strongly decelerated and reached $94 \%$ of its maximum height. In comparison, the bottom part of the arch filament only rises to a height of about $5.4 \mathrm{Mm}$. The velocity in the accelerating phase was determined by taking the derivative of a fit to the height-time profile using a Boltzmann-Sigmoid function of the form $h_{a}(t)=a_{0}+\left(a_{1}-a_{0}\right) /\left(1+e^{\left(a_{2}-t\right) / a_{3}}\right)$ (thick red line). The deceleration phase was fitted with a simple exponential decay curve of the form $h_{d}(t)=b_{0}\left(1-b_{1} e^{\left(-b_{2} t\right)}\right)$ (dashed red line) and the corresponding velocity is shown by cross symbols. The blue symbols represent the deceleration of the filament which is nearly 7.5 times greater than the solar gravitational constant. The strong decelerating force prevents the erupting filament from becoming a CME. Only two events were reported in the CME catalog provided by CDAW. One was a Halo event that occurred at 20:36:05 UT which had a linear speed of $919 \mathrm{~km} / \mathrm{s}$ and originated from an erupting prominence close to the eastern limb $\left(-790^{\prime \prime}, 305^{\prime \prime}\right)$. The other entry in the catalog was a very poor event that occurred much earlier at 09:48:05 UT and was only seen in $\mathrm{C} 2$.

\subsection{Confinement of the C1.1 flare}

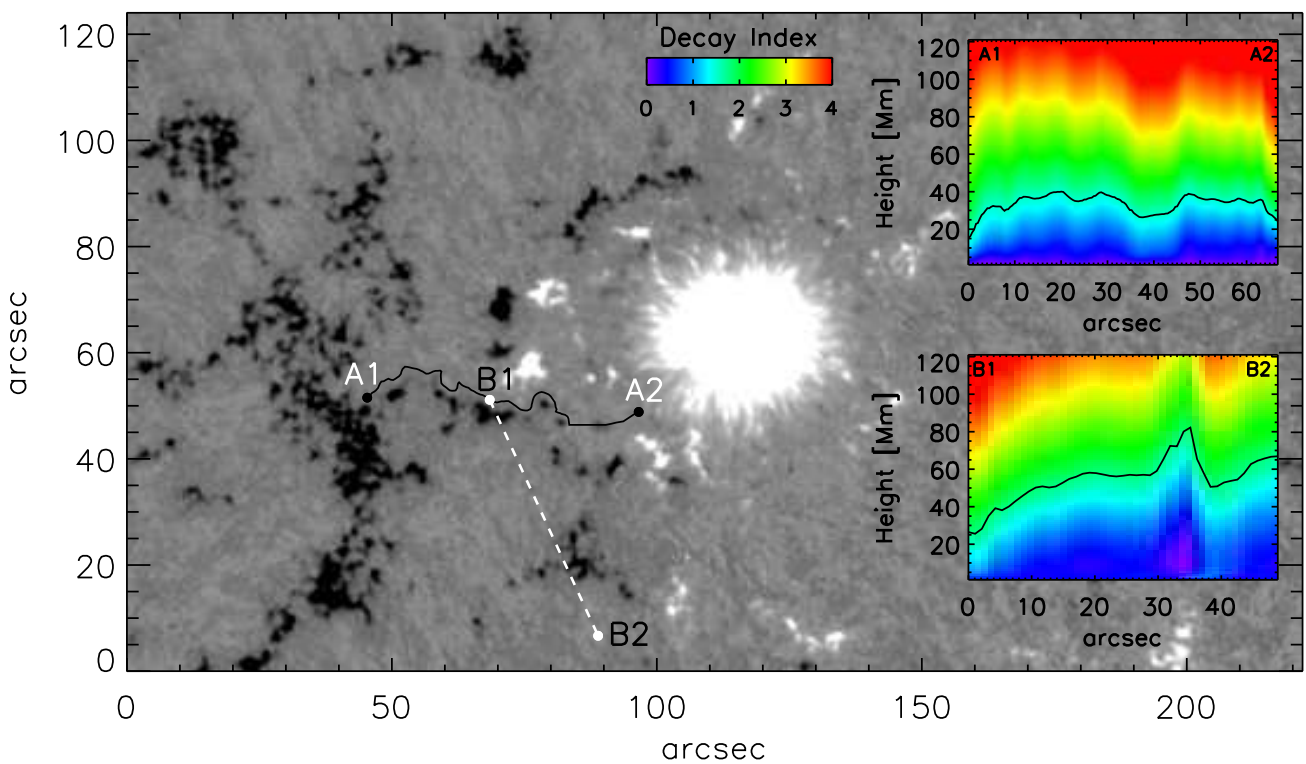

Figure 8. Confinement of the eruption using the decay index. The background image is the vertical component of the magnetic field at 22:36 UT. A1-A2 and B1-B2 are two cuts for which the decay index is shown in the insets on the right. A1-A2 traces the weak PIL under the arch filament, while B1-B2 is along the direction where the filament expands during the C1.1 flare. The black line in the insets corresponds to a value of 1.5 for the decay index.

I further analyze the conditions that led to the initial eruption of the arch filament and its subsequent confinement by computing the decay index from a potential field extrapolation (Cheng, Zhang, Ding, Guo, \& Su, 2011; Jing et al., 2018; Xu, Liu, Jing, \& Wang, 2012) of the vertical component of the magnetic field prior to the C1.1 flare. The decay index is a measure of how fast the horizontal component of the overlying strapping fields changes with height. Figure 9 shows the weak PIL with A1-A2 denoting its span. The decay index $n$ is calculated over this contour as well as along the line B1-B2 which is the direction in which the arch filament unwinds and expands. The insets to the right of the figure show $n$ as a function of height over the two chosen spatial cuts. For the onset of the torus instability (Kliem \& Török, 2006), $n$ has a critical value of 1.5, i.e. when the strapping fields can no 
longer confine the flux rope. The black line in the upper inset indicates that the height at which $n=1.5$ only reaches a maximum value of $40 \mathrm{Mm}$, and reduces to about 25-30 Mm near the central section of the arch filament (near B1). This indicates that the field decays faster at the PIL and cannot confine the arch filament from erupting (Cui et al., 2018; Jing et al., 2015). On the other hand, the iso-contour of $n=1.5$ is a lot higher across the cut B1-B2 which increases southwards reaching a peak value of $85 \mathrm{Mm}$ which is close to the patch of negative polarity about $15^{\prime \prime}$ north-east of B2.

It is evident from the above that the overlying fields across the weak PIL, which decay faster, are unable to confine the arch filament and reconnect as the latter rises, thereby producing the two-ribbon flare. However, the second set of overlying fields connecting the leading sunspot to the network flux region, decay much more slowly and are able to prevent the eruption from developing into a CME.
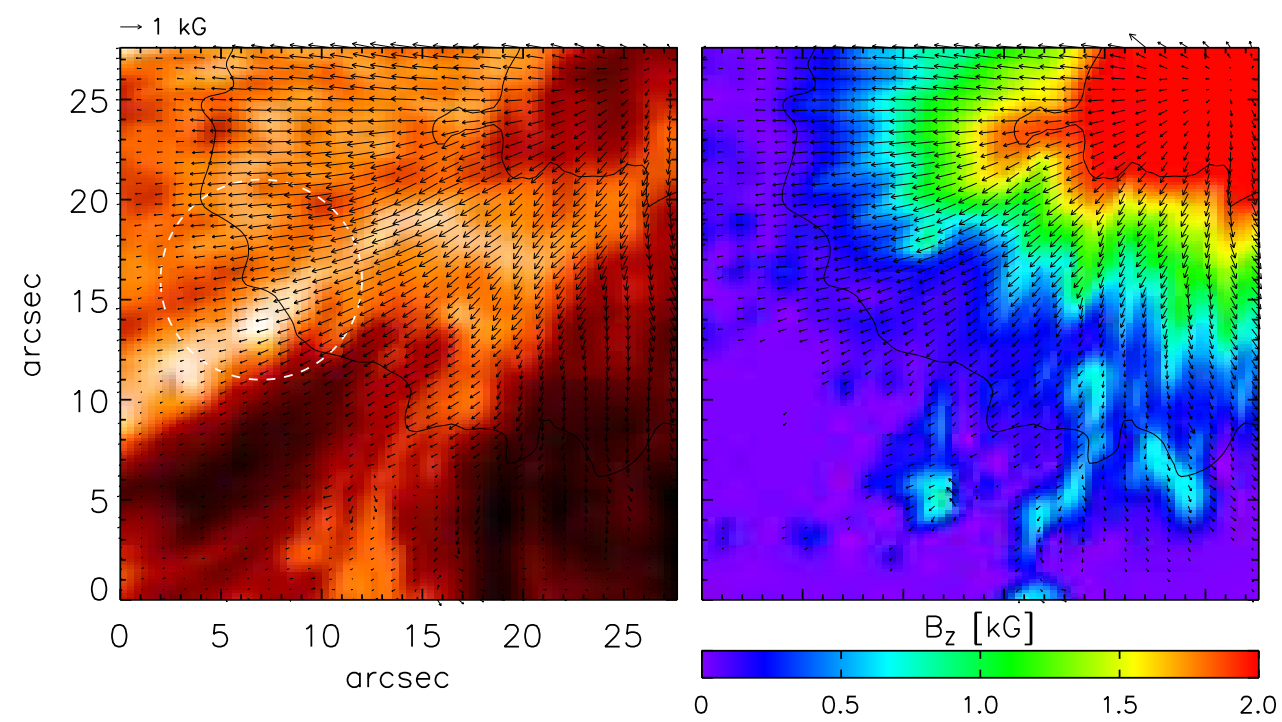

Figure 9. Vector magnetic field derived from TIP-II observations. The arrows correspond to the horizontal magnetic field derived from inversions of the Fe and Si line shown in the left and right panels, respectively. The arrows have been drawn for every third pixel. The background images correspond to the AIA $304 \AA$ image (left) and the vertical component of the magnetic field from the Si line inversions (right), whose scaling is shown in the color bar below. The dashed white circle in the left panel indicates the location of the arch filament F1 anchored to the leading sunspot's boundary.

\subsection{Underlying photospheric conditions}

\subsubsection{Vector magnetic field of the leading sunspot}

In addition to being the strongest and largest concentration of magnetic flux in the AR, the leading sunspot also has an important role to play in initiation of the flare as the underlying photospheric conditions dictate the overlying chromospheric and coronal conditions. This makes it important to determine if there exist any signatures in the photospheric magnetic field of the leading sunspot that could possibly be related to the filament F1 given that one of its footpoints is rooted at the boundary of the spot. Even though the spectropolarimetric observations were acquired nearly $13 \mathrm{hr}$ prior to the C1.1 flare, the filament did not exhibit any significant morphological changes and thus, a comparison with the photospheric 
magnetic field is adequate. Figure 9 shows the horizontal magnetic field derived from the inversions of the $\mathrm{Fe}$ and $\mathrm{Si}$ lines. The left panel shows that the field is predominantly radial and varies smoothly in the azimuthal direction. The white dashed circle indicates the location of F1 at the sunspot boundary (dark filament above a lateral brightening). Using the azimuth value at a location on the umbra-penumbra boundary, a line is traced outwards till it meets F1 at the sunspot boundary. The azimuth value at this location deviated by about $20^{\circ}$ from that at the umbra-penumbra boundary. In addition, the deviation of the horizontal field from the ideal radial direction as seen from above is clockwise, which is consistent with the sign of the twist seen in F1 (Fig. 6). However, more detailed modeling is necessary to confirm if this deviation in the photosphere is reflected in the structure of the overlying arch filament. The right panel of Fig. 9 shows that in the southern quadrant of the sunspot, the vertical component of the magnetic field shows disruptions or patches of weaker field that extend out of the spot's boundary. These patches are related to the dark fibrils that further connect to the large dark channel seen in the AIA images.
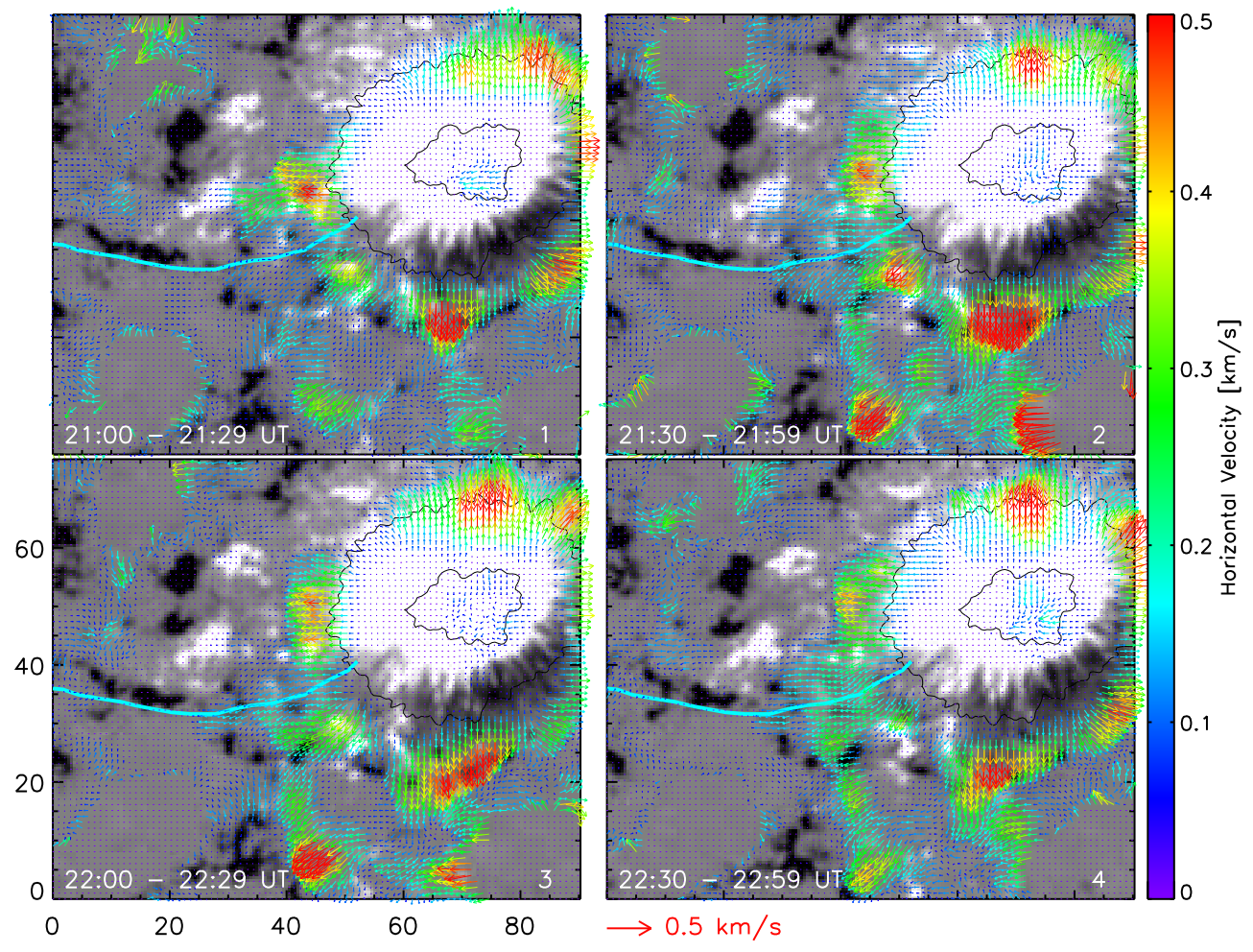

Figure 10. Horizontal flow maps derived from LCT overlaid on the HMI LOS magnetograms. Each panel is an average of the horizontal flows over a $30 \mathrm{~min}$ time span. The background image is a LOS magnetogram averaged over the duration indicated in white in the bottom left corner of the panel. The thick line in cyan represents the arch filament $\mathrm{F} 1$. The $x$ - and $y$-axes are in units of arcsec. The arrows have been shown for every alternate pixel. The colored arrows represent the horizontal speed which are indicated in the color bar on the right.

\subsubsection{Sunspot moat flow driving magnetic flux cancellation}

As seen in earlier in Sects. 3.2.1 and 3.2.2, small-scale flux cancellation occurs at the weak PIL directly beneath the filament F1 which drives the precursor flares. In this section, the nature of the horizontal flows in the vicinity of F1 and the leading sunspot are analyzed to determine the manner and extent to which flux cancellation is facilitated, and the possible role of the moat flow (Brickhouse \& Labonte, 1988; Sheeley, 1972) originating from the spot. 
In order to determine the horizontal flows in the AR, I use local correlation tracking (LCT; Fisher \& Welsch, 2008; November, 1986; November \& Simon, 1988; Welsch, Fisher, Abbett, \& Regnier, 2004). LCT computes the relative displacement of small sub-sections in an image centered on the pixel of interest. A Gaussian window of a certain full-width-at-halfmaximum (FWHM), that is about the size of the structures that are to be tracked, is used to apodize the sub-images. The horizontal speed can thus be determined at every pixel given the displacement and the time separation. In order to extract the two-dimensional flow, LCT is applied to the HMI LOS magnetogram sequence. The magnetograms are first smoothed using a $7 \times 7$ pixel boxcar and then subtracted from the original image so that the small-scale magnetic fragments can be tracked easily. This scheme is similar to the one adopted by Ravindra, Venkatakrishnan, and Kumar (2004). An apodizing window with a $\sigma=7$ pixels is used for the LCT routine. Although the time separation is $45 \mathrm{~s}$, the final horizontal flow field is obtained by averaging 40 images to retain the persistent motions and suppress the noise.

Figure 10 shows the horizontal flows derived from LCT averaged over a 30 min interval leading upto the $\mathrm{C} 1.1$ flare. Moat flows ranging between 0.3 and $0.5 \mathrm{~km} \mathrm{~s}^{-1}$ are observed outside the sunspot extending to about 10-15" outside the visible spot boundary. While the moat flow is nearly uniform around the sunspot's circumference, there is a region near the north-east quadrant where the flow is inhibited. This could be attributed to the presence of a large, magnetic fragment of positive polarity just outside the spot boundary and well within the moat radius, which obstructs the outward flow. This is in agreement with the observations of Vargas Domínguez et al. (2008). The moat flow also persists for a period of $2 \mathrm{hrs}$ which reflect the continuous outflow from the sunspot that is functional on longer timescales. In general, the figure indicates that the horizontal flow is greatly reduced in the neighborhood of pre-existing flux that do not exhibit any proper motion, but rather, evolve in-situ.

The outward flow from the sunspot, particularly in the south-eastern quadrant is also directed along the axis representing the filament F1 which is shown in cyan. These flows which are about $0.2-0.3 \mathrm{~km} \mathrm{~s}^{-1}$ in magnitude extend upto a distance of about $20^{\prime \prime}$ into F1. The flux cancellation event associated with the first precursor flare is aided by a horizontal flow of about $0.2 \mathrm{~km} \mathrm{~s}^{-1}$ that is directed from west to east. The flows reduce considerably once they encounter the large, pre-existing flux patch of negative polarity which lies underneath the central part of F1 and is evident from panel 2 of the figure. In the case of the second precursor flare, the flux cancellation occurs $10^{\prime \prime}$ further east where the flows are considerably weaker till 22:30 UT, with values of about $0.1 \mathrm{~km} \mathrm{~s}^{-1}$ (panel 3). However, during the course of the $\mathrm{C} 1.1$ flare, the flows increase to $0.2 \mathrm{~km} \mathrm{~s}^{-1}$ at this location as seen in panel 4 of the figure. The figure also shows that the horizontal flows at the weak PIL (region around $x=20^{\prime \prime}, y=35^{\prime \prime}$ ) are strongly inhibited, which could be attributed to magneto-convection and remain unaffected by the spot's moat flow. Horizontal flows of about $0.5 \mathrm{~km} \mathrm{~s}^{-1}$ are also seen to be directed into the dark channel (marked in yellow in Fig. 2) that starts about 20-25" from the southern boundary of the sunspot.

\section{Discussion}

A C1.1 class, confined flare is analyzed in this article that occurred in an arch filament system very close to a regular, unipolar, isolated sunspot. While the continuum images do not show any distinguishable presence of a following polarity of the active region, the LOS magnetograms reveal a rather diffuse, but extended network flux patch. Chromospheric images from AIA indicate that there are three arch filaments, rooted in the following polarity with the two larger ones extending upto the boundary of the leading sunspot. In addition to the arch filaments, there are overlying hotter loops connecting the two major polarities of the AR. The flare occurred in the southern arch filament. Spectro-polarimetric observations from the TIP-II instrument at the $70 \mathrm{~cm} \mathrm{VTT}$, show a small deviation of the horizontal magnetic field from a radial orientation close to the sunspot's boundary where the arch 
filament is oriented. The sense of the deviation of the magnetic field is consistent with the handedness of the arch filament although detailed modeling is necessary to substantiate this. The PIL between the leading sunspot and the network flux region, although devoid of any complex magnetic structures, nevertheless comprises a few, small, magnetic fragments of mixed polarity. One such set of fragments forms a very weak PIL that is located directly beneath the arch filament.

The C1.1 flare is preceded by two, small-scale flaring events that occur within an hour of the former. The first event comprises a set of loops that expand rapidly and detach from the arch filament producing brightenings at the loop footpoints on either side of the weak PIL underlying the arch filament. This precursor flare is similar to the event described in H. Wang et al. (2015). The other precursor event, which occurs about 30 min later, also produces similar, but stronger, footpoint brightenings at/close to the same location as the earlier event. A section of the arch filament is filled by cool plasma immediately after the precursor flare that is often seen after an eruption (Gilbert, Holzer, \& Burkepile, 2001; Gilbert, Holzer, Burkepile, \& Hundhausen, 2000; R. Liu, Alexander, \& Gilbert, 2007; Louis, Balthasar, et al., 2014). Following the precursor flaring events, the arch filament is seen to exhibit an increased number of intersections of bright and dark threads suggesting an increase in its twist. The C1.1 flare involves a fast rise and expansion of the arch filament while also producing a two-ribbon emission at the weak PIL. The flare results in the formation of a more relaxed arch connecting the two polarities of the active region. The flare emission is located close to the centroid of the active region which is in good agreement with the estimates provided by Y. Wang and Zhang (2007) and Cheng et al. (2011) for confined flares.

The photospheric driver of the precursor events, leading upto the C1.1 flare, is smallscale flux cancellation at the weak PIL underlying the arch filament. The flux loss rate is one to two orders of magnitude smaller than those associated with surges and EUV jets (Y. C. Jiang, Chen, Li, Shen, \& Yang, 2007; Y. Liu \& Kurokawa, 2004). Nevertheless, flux cancellation is co-spatial as well as co-temporal with the precursor flare brightenings and there are no visible or detectable signatures of flux emergence occurring in the region close to the arch filament. One also finds that the moat flow from the sunspot aids in flux cancellation, particularly near the end of the arch filament that is rooted at the boundary of the sunspot. The moat flow is responsible for pushing small magnetic elements into the path of pre-existing patches of opposite polarity.

It is known that flux cancellation can lead to the accumulation of helicity in the corona (Amari, Luciani, Mikic, \& Linker, 2000; Burtseva \& Petrie, 2013; Green, Kliem, \& Wallace, 2011; Linker, Lionello, Mikić, \& Amari, 2001; Livi, Martin, Wang, \& Ai, 1989; Mathew \& Ambastha, 2000; Savcheva, Green, van Ballegooijen, \& DeLuca, 2012; Sterling, Chifor, Mason, Moore, \& Young, 2010; Sterling et al., 2007; van Ballegooijen \& Martens, 1989; H. Wang et al., 2017; Zhang, Wang, Deng, \& Wu, 2001). Flux cancellation at the PIL under the arch filament produce the precursor flaring events and introduce more twist in the arch filament, eventually destabilizing it (Guo, Ding, Cheng, Zhao, \& Pariat, 2013; Hood \& Priest, 1981; R. Liu, Gilbert, Alexander, \& Su, 2008; Xue et al., 2016) through the helical kink instability (Török, Kliem, \& Titov, 2004). While an extrapolation of the photospheric magnetic field into the overlying corona is necessary to determine the amount of twist in the arch filament following the precursor activities, one can qualitatively determine the twist through the number of dark and bright windings on the filament as shown in Fig. 6. One can visualize at least two dark threads which would suggest that the number of turns in the filament would be 1 or $2 \pi$ radian. This lower bound value of the twist is indicative of a weakly twisted, low-lying, flux rope (Chintzoglou, Patsourakos, \& Vourlidas, 2015). In an alternate scenario, flux ropes can also be formed during strong, confined flares quickly leading upto a major CME within a short duration of time (L. Liu, Cheng, et al., 2018).

The two-ribbon flare is the result of the unstable arch filament rising, causing the overlying fields across the weak PIL to reconnect. The overlying fields connecting the major 
polarities of the active region, which decay much more slowly, exert a strong tension force in the downward direction which brakes the erupting arch filament, causing it to decelerate (Török \& Kliem, 2005). This is evident from Fig. 7 where the deceleration exceeds the solar gravitational constant by a factor of nearly 8 . The height-time profile of the rising filament is similar to the findings of Ji, Wang, Schmahl, Moon, and Jiang (2003) for a confined filament eruption where the filament reached a maximum height of about $80 \mathrm{Mm}$ and a speed of about $200 \mathrm{~km} \mathrm{~s}^{-1}$. The height and speed with which the filament rises is dependent on the strength of the axial magnetic field and the twist of the filament (Archontis \& Török, 2008; Murray, Hood, Moreno-Insertis, Galsgaard, \& Archontis, 2006). Furthermore, being a weak flare of C1.1 class, there likely would have been a reduced Lorentz force impulse at the onset of eruption to overcome the tension force from the higher overlying fields (L. Liu, Wang, et al., 2018).

Following the flare, the arch filament relaxes to a simple loop-like structure as seen in panels 9 and 10 of Fig. 6. The loss of twist could be attributed to magnetic reconnection occurring in either of the following scenarios. Firstly, the filament could have underwent internal reconnection, arising from the buckling of the filament axis on the western leg that is evident during the peak of the flare (panel 6 of Fig. 6). This distortion of the axis likely brings one part of the filament close to another section allowing magnetic reconnection and thus removing the overall twist of the filament. The alternate scenario is that, as the filament rises the outermost set of twisted field lines reconnect with the lowermost section of the overlying fields connecting the two major polarities of the active region. Although the axial field has the same direction for both sets of field lines, it could be that certain sections of the filament have a component of the magnetic field where reconnection could be initiated. In either of the above cases, one observes elongated brightenings along the upper part of the western half and the lower part of the eastern half of the arch filament (panel 7 of Fig. 6) which suggest that reconnection is responsible for the loss of twist in the filament rendering a simple potential configuration at the end of the flare.

The active region did produce two additional flares along with the C1.1 flare on September 24, which included a C3.9 flare on September 18 and a B4.8 flare on September 23. Interestingly, the C3.9 flare was the only one out of the three flares, which produced a CME, but the active region was just moving across the eastern limb of the Sun at that time. The CME had a linear speed of $208 \mathrm{~km} \mathrm{~s}^{-1}$ and an angular width of $52^{\circ}$. However, the active region was still an $\alpha$-type at that time and remained so for the entirety of its passage across the disc. Hence, it would be interesting to investigate what facilitated the eruption into an ensuing CME given that the photospheric magnetic conditions were similar to the event described here where the eruption remained confined.

\section{Conclusion}

A C1.1 class, confined flare is investigated in this article which occurred on 2013 September 24 at 22:56 UT, in an arch filament system close to a regular, unipolar sunspot. The legs of this arch filament were anchored in the leading sunspot of positive polarity and the network flux region of negative polarity. The flare was driven by small-scale flux cancellation at the weak PIL underlying the arch filament which resulted in two, small flaring events within an hour of the C1.1 flare. Flux cancellation was aided by the moat flow from the leading sunspot which was also one of the anchorage points of the arch filament. The cancellation of flux led to the destabilization of the arch filament which was seen as an increase in the twist along the arch filament. The horizontal fields across the weak PIL decay faster which cannot prevent the filament from rising which resulted in a two-ribbon flare at the PIL. On the other hand, the overlying fields between the two polarities of the active region, decay much more slowly and exert a strong downward directed tension force which decelerates the rising filament and prevents it from developing into a CME. It would be worthwhile to investigate if the above photospheric and coronal conditions are generic to flares occurring in simple, bipolar active regions. 


\section{Acknowledgments}

The Vacuum Tower Telescope is operated by the Kiepenheuer-Institute for Solar Physics in Freiburg, Germany, at the Spanish Observatorio del Teide, Tenerife, Canary Islands. The observing campaign at the Vacuum Tower Telescope was supported by the German Science Foundation (DFG) under grant DE 787/3-1. HMI data is courtesy of NASA/SDO and the HMI science team. They are provided by the Joint Science Operations Center - Science Data Processing at Stanford University. This work was (partly) carried out by using Hinode Flare Catalogue (https://hinode.isee.nagoya-u.ac.jp/flarel_catalogue/), which is maintained by ISAS/JAXA and Institute for Space-Earth Environmental Research (ISEE), Nagoya University. This work made use of the SOHO LASCO CME Catalog which is generated and maintained at the CDAW Data Center by NASA and The Catholic University of America in cooperation with the Naval Research Laboratory. SOHO is a project of international cooperation between ESA and NASA. REL is grateful to the reviewers for their useful comments which significantly improved the article.

\section{References}

Amari, T., Luciani, J. F., Mikic, Z., \& Linker, J. (2000, Jan). A Twisted Flux Rope Model for Coronal Mass Ejections and Two-Ribbon Flares. Astrophys. J., 529(1), L49-L52. doi: $10.1086 / 312444$

Archontis, V., \& Török, T. (2008, Dec). Eruption of magnetic flux ropes during flux emergence. Astron. Astrophys., 492(2), L35-L38. doi: 10.1051/0004-6361:200811131

Balthasar, H., \& Gömöry, P. (2008, September). The three-dimensional structure of sunspots. I. The height dependence of the magnetic field. Astron. Astrophys., 488, 1085-1092. doi: 10.1051/0004-6361:200809575

Brickhouse, N. S., \& Labonte, B. J. (1988, March). Mass and energy flow near sunspots. I - Observations of moat properties. Sol. Phys., 115, 43-60. doi: 10.1007/BF00146229

Bruzek, A. (1967, Dec). On Arch-Filament Systems in Spotgroups. Sol. Phys., 2(4), 451-461. doi: 10.1007/BF00146493

Burtseva, O., \& Petrie, G. (2013, April). Magnetic Flux Changes and Cancellation Associated with X-Class and M-Class Flares. Sol. Phys., 283, 429-452. doi: 10.1007/ s11207-013-0241-8

Chae, J. (2000, September). The Magnetic Helicity Sign of Filament Chirality. Astrophys. J., 540, L115-L118. doi: 10.1086/312880

Chen, H., Zhang, J., Ma, S., Yang, S., Li, L., Huang, X., \& Xiao, J. (2015, July). Confined Flares in Solar Active Region 12192 from 2014 October 18 to 29. Astrophys. J., 808, L24. doi: 10.1088/2041-8205/808/1/L24

Cheng, X., Zhang, J., Ding, M. D., Guo, Y., \& Su, J. T. (2011, May). A Comparative Study of Confined and Eruptive Flares in NOAA AR 10720. Astrophys. J., 732, 87. doi: $10.1088 / 0004-637 \mathrm{X} / 732 / 2 / 87$

Chintzoglou, G., Patsourakos, S., \& Vourlidas, A. (2015, Aug). Formation of Magnetic Flux Ropes during a Confined Flaring Well before the Onset of a Pair of Major Coronal Mass Ejections. The Astrophysical Journal, 809(1), 34. doi: 10.1088/0004-637X/809/1/34

Collados, M. (1999, September). High Resolution Spectropolarimetry and Magnetography. In B. Schmieder, A. Hofmann, \& J. Staude (Eds.), Third advances in solar physics euroconference: Magnetic fields and oscillations (Vols. ASP Conf. Ser., 184, p. 3-22).

Collados, M., Lagg, A., Díaz Garcí A, J. J., Hernández Suárez, E., López López, R., Páez Mañá, E., \& Solanki, S. K. (2007, May). Tenerife Infrared Polarimeter II. In P. Heinzel, I. Dorotovič, \& R. J. Rutten (Eds.), The physics of chromospheric plasmas (Vols. ASP Conf. Ser., 368, p. 611).

Cui, Y., Wang, H., Xu, Y., \& Liu, S. (2018, March). Statistical Study of Magnetic Topology for Eruptive and Confined Solar Flares. J. Geophys. Res. (Space Physics), 123, 17041714. doi: 10.1002/2017JA024710

Fisher, G. H., \& Welsch, B. T. (2008). FLCT: A Fast, Efficient Method for Performing Local Correlation Tracking. In R. Howe, R. W. Komm, K. S. Balasubramaniam, 
\& G. J. D. Petrie (Eds.), Subsurface and atmospheric influences on solar activity (Vol. 383, p. 373).

Forbes, T. (2010). Models of coronal mass ejections and flares. In C. J. Schrijver \& G. L. Siscoe (Eds.), Heliophysics: Space storms and radiation: Causes and effects (p. 159). Cambridge University Press.

Foukal, P. (1971, Aug). Morphological Relationships in the Chromospheric H $\alpha$ Fine Structure. Sol. Phys., 19(1), 59-71. doi: 10.1007/BF00148824

Gilbert, H. R., Holzer, T. E., \& Burkepile, J. T. (2001, Mar). Observational Interpretation of an Active Prominence on 1999 May 1. Astrophys. J., 549(2), 1221-1230. doi: $10.1086 / 319444$

Gilbert, H. R., Holzer, T. E., Burkepile, J. T., \& Hundhausen, A. J. (2000, Jul). Active and Eruptive Prominences and Their Relationship to Coronal Mass Ejections. Astrophys. J., 537(1), 503-515. doi: 10.1086/309030

Gosling, J. T. (1993, Jul). Coronal mass ejections: The link between solar and geomagnetic activity. Physics of Fluids B, 5(7), 2638-2645. doi: 10.1063/1.860701

Green, L. M., Kliem, B., \& Wallace, A. J. (2011, February). Photospheric flux cancellation and associated flux rope formation and eruption. Astron. Astrophys., 526, A2. doi: $10.1051 / 0004-6361 / 201015146$

Guo, Y., Ding, M. D., Cheng, X., Zhao, J., \& Pariat, E. (2013, Dec). Twist Accumulation and Topology Structure of a Solar Magnetic Flux Rope. The Astrophysical Journal, $779(2), 157$. doi: $10.1088 / 0004-637 \mathrm{X} / 779 / 2 / 157$

Hagyard, M. J., Venkatakrishnan, P., \& Smith, J. B., Jr. (1990, June). Nonpotential magnetic fields at sites of gamma-ray flares. Astrophys. J. (Supp.),, 73, 159-163. doi: $10.1086 / 191447$

Hood, A. W., \& Priest, E. R. (1981, Oct). Thermal Nonequilibrium - a Trigger for Solar Flares. Sol. Phys., 73(2), 289-311. doi: 10.1007/BF00151683

Huang, Z., Mou, C., Fu, H., Deng, L., Li, B., \& Xia, L. (2018, Feb). A Magnetic Reconnection Event in the Solar Atmosphere Driven by Relaxation of a Twisted Arch Filament System. Astrophys. J., 853(2), L26. doi: 10.3847/2041-8213/aaa88c

Ji, H., Wang, H., Schmahl, E. J., Moon, Y. J., \& Jiang, Y. (2003, Oct). Observations of the Failed Eruption of a Filament. Astrophys. J., 595(2), L135-L138. doi: 10.1086/378178

Jiang, C., Wu, S. T., Yurchyshyn, V., Wang, H., Feng, X., \& Hu, Q. (2016, September). How Did a Major Confined Flare Occur in Super Solar Active Region 12192? Astrophys. J., 828, 62. doi: 10.3847/0004-637X/828/1/62

Jiang, Y. C., Chen, H. D., Li, K. J., Shen, Y. D., \& Yang, L. H. (2007, Jul). The H $\alpha$ surges and EUV jets from magnetic flux emergences and cancellations. Astron. Astrophys., 469(1), 331-337. doi: 10.1051/0004-6361:20053954

Jing, J., Liu, C., Lee, J., Ji, H., Liu, N., Xu, Y., \& Wang, H. (2018, Sep). Statistical Analysis of Torus and Kink Instabilities in Solar Eruptions. Astrophys. J., 864(2), 138. doi: $10.3847 / 1538-4357 /$ aad6e4

Jing, J., Xu, Y., Lee, J., Nitta, N. V., Liu, C., Park, S.-H., .. Wang, H. (2015, September). Comparison between the eruptive X2.2 flare on 2011 February 15 and confined X3.1 flare on 2014 October 24. Research in Astronomy and Astrophysics, 15, 1537. doi: 10.1088/1674-4527/15/9/010

Jing, J., Yurchyshyn, V. B., Yang, G., Xu, Y., \& Wang, H. (2004, Oct). On the Relation between Filament Eruptions, Flares, and Coronal Mass Ejections. Astrophys. J., 614 (2), 1054-1062. doi: 10.1086/423781

Kahler, S. W. (1992, Jan). Solar flares and coronal mass ejections. ARA 6 A, 30, 113-141. doi: 10.1146/annurev.aa.30.090192.000553

Karpen, J. T., Antiochos, S. K., \& DeVore, C. R. (2012, Nov). The Mechanisms for the Onset and Explosive Eruption of Coronal Mass Ejections and Eruptive Flares. Astrophys. J., 760(1), 81. doi: 10.1088/0004-637X/760/1/81

Kliem, B., \& Török, T. (2006, June). Torus Instability. Phys. Rev. Lett., 96 (25), 255002. doi: 10.1103/PhysRevLett.96.255002

Lemen, J. R., Title, A. M., Akin, D. J., Boerner, P. F., Chou, C., Drake, J. F., .. Waltham, 
N. (2012, January). The Atmospheric Imaging Assembly (AIA) on the Solar Dynamics Observatory (SDO). Sol. Phys., 275, 17-40. doi: 10.1007/s11207-011-9776-8

Linker, J. A., Lionello, R., Mikić, Z., \& Amari, T. (2001, Nov). Magnetohydrodynamic modeling of prominence formation within a helmet streamer. J. Geophys. Res., 106(A11), 25165-25176. doi: 10.1029/2000JA004020

Liu, L., Cheng, X., Wang, Y., Zhou, Z., Guo, Y., \& Cui, J. (2018, Nov). Rapid Buildup of a Magnetic Flux Rope during a Confined X2.2 Class Flare in NOAA AR 12673. The Astrophysical Journal, 867(1), L5. doi: 10.3847/2041-8213/aae826

Liu, L., Wang, Y., Wang, J., Shen, C., Ye, P., Liu, R., .. Wang, S. (2016, August). Why is a Flare-rich Active Region CME-poor? Astrophys. J., 826, 119. doi: 10.3847/ 0004-637X/826/2/119

Liu, L., Wang, Y., Zhou, Z., Dissauer, K., Temmer, M., \& Cui, J. (2018, May). A Comparative Study between a Failed and a Successful Eruption Initiated from the Same Polarity Inversion Line in AR 11387. Astrophys. J., 858(2), 121. doi: $10.3847 / 1538-4357 /$ aabba2

Liu, R., Alexander, D., \& Gilbert, H. R. (2007, Jun). Kink-induced Catastrophe in a Coronal Eruption. Astrophys. J., 661(2), 1260-1271. doi: 10.1086/513269

Liu, R., Gilbert, H. R., Alexander, D., \& Su, Y. (2008, Jun). The Effect of Magnetic Reconnection and Writhing in a Partial Filament Eruption. Astrophys. J., 680(2), 1508-1515. doi: $10.1086 / 587482$

Liu, Y., \& Kurokawa, H. (2004, Aug). On a Surge: Properties of an Emerging Flux Region. Astrophys. J., 610(2), 1136-1147. doi: 10.1086/421715

Livi, S. H. B., Martin, S., Wang, H., \& Ai, G. (1989, March). The association of flares to cancelling magnetic features on the sun. Sol. Phys., 121, 197-214. doi: 10.1007/ BF00161696

Louis, R. E., Balthasar, H., Kuckein, C., Gömöry, P., Puschmann, K. G., \& Denker, C. (2014, February). The association between sunspot magnetic fields and superpenumbral fibrils. Astronomische Nachrichten, 335, 161. doi: 10.1002/asna.201312017

Louis, R. E., Beck, C., \& Ichimoto, K. (2014, July). Small-scale chromospheric jets above a sunspot light bridge. Astron. Astrophys., 567, A96. doi: 10.1051/0004-6361/ 201423756

Martin, S. F. (1998a, Sep). Conditions for the Formation and Maintenance of Filaments (Invited Review). Sol. Phys., 182(1), 107-137. doi: 10.1023/A:1005026814076

Martin, S. F. (1998b). Filament Chirality: A Link Between Fine-Scale and Global Patterns (Review). In D. F. Webb, B. Schmieder, \& D. M. Rust (Eds.), Iau colloq. 167: New perspectives on solar prominences (Vols. ASP Conf. Ser., 150, p. 419).

Martin, S. F. (2003). Signs of helicity in solar prominences and related features. Adv. Sp. Res., 32, 1883-1893. doi: 10.1016/S0273-1177(03)90622-3

Mathew, S. K., \& Ambastha, A. (2000, November). Magnetic field gradient and flare: study of a small flare in NOAA 8038. Sol. Phys., 197, 75-84. doi: 10.1023/A:1026568430463

Murray, M. J., Hood, A. W., Moreno-Insertis, F., Galsgaard, K., \& Archontis, V. (2006, Dec). 3D simulations identifying the effects of varying the twist and field strength of an emerging flux tube. Astron. Astrophys., 460(3), 909-923. doi: 10.1051/0004-6361: 20065950

November, L. J. (1986, February). Measurement of geometric distortion in a turbulent atmosphere. Appl. Opt., 25, 392-397. doi: 10.1364/AO.25.000392

November, L. J., \& Simon, G. W. (1988, October). Precise proper-motion measurement of solar granulation. Astrophys. J., 333, 427-442. doi: 10.1086/166758

Plunkett, S. P., Vourlidas, A., Šimberová, S., Karlický, M., Kotrč, P., Heinzel, P., ... Wu, S. T. (2000, Jun). Simultaneous SOHO and Ground-Based Observations of a Large Eruptive Prominence and Coronal Mass Ejection. Sol. Phys., 194(2), 371-391. doi: 10.1023/A:1005287524302

Ravindra, B., Venkatakrishnan, P., \& Kumar, B. (2004, November). The Pattern of Moving Magnetic Inhomogeneities in and Around Sunspots. Sol. Phys., 225, 47-58. doi: 10.1007/s11207-004-1616-7 
Ruiz Cobo, B., \& del Toro Iniesta, J. C. (1992, October). Inversion of Stokes profiles. Astrophys. J., 398, 375-385. doi: 10.1086/171862

Sarkar, R., \& Srivastava, N. (2018, February). A Comparative Study of the Eruptive and Non-eruptive Flares Produced by the Largest Active Region of Solar Cycle 24. Sol. Phys., 293, 16. doi: 10.1007/s11207-017-1235-8

Savcheva, A. S., Green, L. M., van Ballegooijen, A. A., \& DeLuca, E. E. (2012, November). Photospheric Flux Cancellation and the Build-up of Sigmoidal Flux Ropes on the Sun. Astrophys. J., 759, 105. doi: 10.1088/0004-637X/759/2/105

Schou, J., Scherrer, P. H., Bush, R. I., Wachter, R., Couvidat, S., Rabello-Soares, M. C., ... Tomczyk, S. (2012, January). Design and Ground Calibration of the Helioseismic and Magnetic Imager (HMI) Instrument on the Solar Dynamics Observatory (SDO). Sol. Phys., 275, 229-259. doi: 10.1007/s11207-011-9842-2

Schrijver, C. J. (2009, March). Driving major solar flares and eruptions: A review. Advances in Space Research, 43, 739-755. doi: 10.1016/j.asr.2008.11.004

Sheeley, N. R., Jr. (1972, July). Observations of the Horizontal Velocity Field Surrounding Sunspots. Sol. Phys., 25, 98-103. doi: 10.1007/BF00155747

Sterling, A. C., Chifor, C., Mason, H. E., Moore, R. L., \& Young, P. R. (2010, October). Evidence for magnetic flux cancelation leading to an ejective solar eruption observed by Hinode, TRACE, STEREO, and SoHO/MDI. Astron. Astrophys., 521, A49. doi: 10.1051/0004-6361/201014006

Sterling, A. C., Moore, R. L., Berger, T. E., Bobra, M., Davis, J. M., Jibben, P., .. Weber, M. (2007, Nov). Hinode Observations of the Onset Stage of a Solar Filament Eruption. PASJ, 59, S823. doi: 10.1093/pasj/59.sp3.S823

Su, Y., Liu, R., Li, S., Cao, W., Ahn, K., \& Ji, H. (2018, Mar). High-resolution Observations of Flares in an Arch Filament System. Astrophys. J., 855(2), 77. doi: 10.3847/ $1538-4357 /$ aaac31

Sun, X., Bobra, M. G., Hoeksema, J. T., Liu, Y., Li, Y., Shen, C., ... Fisher, G. H. (2015, May). Why Is the Great Solar Active Region 12192 Flare-rich but CME-poor? Astrophys. J., 804, L28. doi: 10.1088/2041-8205/804/2/L28

Török, T., \& Kliem, B. (2005, September). Confined and Ejective Eruptions of Kinkunstable Flux Ropes. Astrophys. J., 630, L97-L100. doi: 10.1086/462412

Török, T., Kliem, B., \& Titov, V. S. (2004, Jan). Ideal kink instability of a magnetic loop equilibrium. Astron. Astrophys., 413, L27-L30. doi: 10.1051/0004-6361:20031691

van Ballegooijen, A. A., \& Martens, P. C. H. (1989, August). Formation and eruption of solar prominences. Astrophys. J., 343, 971-984. doi: 10.1086/167766

Vargas Domínguez, S., Rouppe van der Voort, L., Bonet, J. A., Martínez Pillet, V., Van Noort, M., \& Katsukawa, Y. (2008, May). Moat Flow in the Vicinity of Sunspots for Various Penumbral Configurations. Astrophys. J., 679, 900-909. doi: 10.1086/587139

von der Lühe, O., Soltau, D., Berkefeld, T., \& Schelenz, T. (2003, February). KAOS: Adaptive optics system for the Vacuum Tower Telescope at Teide Observatory. In S. L. Keil \& S. V. Avakyan (Eds.), Innovative telescopes and instrumentation for solar astrophysics (Vol. 4853, p. 187-193). doi: 10.1117/12.498659

Wang, H. (2006, September). Rapid Changes of Photospheric Magnetic Fields around Flaring Magnetic Neutral Lines. Astrophys. J., 649, 490-497. doi: 10.1086/506320

Wang, H., Cao, W., Liu, C., Xu, Y., Liu, R., Zeng, Z., ... Ji, H. (2015, Apr). Witnessing magnetic twist with high-resolution observation from the 1.6-m New Solar Telescope. Nature Communications, 6, 7008. doi: 10.1038/ncomms8008

Wang, H., Liu, C., Ahn, K., Xu, Y., Jing, J., Deng, N., .. Fleishman, G. D. (2017, Mar). High-resolution observations of flare precursors in the low solar atmosphere. Nature Astronomy, 1, 0085. doi: 10.1038/s41550-017-0085

Wang, Y., Su, Y., Shen, J., Yang, X., Cao, W., \& Ji, H. (2018, Jun). High-resolution He I $10830 \AA$ Narrowband Imaging for an M-class Flare. II. Multiple Hot Channels: Their Origin and Destination. Astrophys. J., 859(2), 148. doi: 10.3847/1538-4357/aac0f7

Wang, Y., \& Zhang, J. (2007, August). A Comparative Study between Eruptive X-Class Flares Associated with Coronal Mass Ejections and Confined X-Class Flares. Astro- 
phys. J., 665, 1428-1438. doi: 10.1086/519765

Weart, S. R., \& Zirin, H. (1969, Jun). The Birth of Active Regions. Publ. A. S. P., 81(480), 270. doi: $10.1086 / 128771$

Welsch, B. T., Fisher, G. H., Abbett, W. P., \& Regnier, S. (2004, August). ILCT: Recovering Photospheric Velocities from Magnetograms by Combining the Induction Equation with Local Correlation Tracking. Astrophys. J., 610, 1148-1156. doi: 10.1086/421767

Xu, Y., Liu, C., Jing, J., \& Wang, H. (2012, Dec). On the Relationship between the Coronal Magnetic Decay Index and Coronal Mass Ejection Speed. Astrophys. J., 761(1), 52. doi: 10.1088/0004-637X/761/1/52

Xue, Z., Yan, X., Cheng, X., Yang, L., Su, Y., Kliem, B., ... Zhao, L. (2016, Jun). Observing the release of twist by magnetic reconnection in a solar filament eruption. Nature Communications, 7, 11837. doi: 10.1038/ncomms11837

Yan, X. L., Qu, Z. Q., \& Kong, D. F. (2011, Jul). Relationship between eruptions of active-region filaments and associated flares and coronal mass ejections. Mon. Not. $R$. Astron. Soc., 414(4), 2803-2811. doi: 10.1111/j.1365-2966.2011.18336.x

Yashiro, S., Akiyama, S., Gopalswamy, N., \& Howard, R. A. (2006, October). Different Power-Law Indices in the Frequency Distributions of Flares with and without Coronal Mass Ejections. Astrophys. J., 650, L143-L146. doi: 10.1086/508876

Zhang, J., Wang, J., Deng, Y., \& Wu, D. (2001, February). Magnetic Flux Cancellation Associated with the Major Solar Event on 2000 July 14. Astrophys. J., 548, L99-L102. doi: $10.1086 / 318934$

Zuccarello, F., Battiato, V., Contarino, L., Guglielmino, S., Romano, P., \& Spadaro, D. (2008, Sep). A C-level flare observed in an arch filament system: reconnection between pre-existing and emerging field lines? Astron. Astrophys., 488(3), 1117-1123. doi: 10.1051/0004-6361:20079171

Zwaan, C. (1985, Oct). The Emergence of Magnetic Flux. Sol. Phys., 100, 397. doi: $10.1007 / \mathrm{BF} 00158438$ 